Article

\title{
Statistical Analysis of Displacement and Length Relation for Normal Faults in the Barents Sea
}

\author{
Dmitriy Kolyukhin ${ }^{1, *}$, Anita Torabi ${ }^{2}\left(\right.$ ) , Audun Libak ${ }^{2}$, Behzad Alaei ${ }^{3}$ and Tatiana Khachkova ${ }^{1}$ \\ 1 Trofimuk Institute of Petroleum Geology and Geophysics SB RAS, pr. Koptyuga, 3, \\ 630090 Novosibirsk, Russia; khachkovats@ipgg.sbras.ru \\ 2 NORCE Norwegian Research Centre, Nygaardsgaten 112, 5008 Bergen, Norway; \\ anto@norceresearch.no (A.T.); audunlibak@gmail.com (A.L.) \\ 3 Earth Science Analytics AS, 4021 Stavanger, Norway; behzad.alaei@earthanalytics.no \\ * Correspondence: KolyukhinDR@ipgg.sbras.ru; Tel.: +7-383-3301337
}

Received: 14 September 2018; Accepted: 12 November 2018; Published: 14 November 2018

check for updates

\begin{abstract}
This paper is devoted to the statistical analysis of dependence between fault length (L) and displacement (D). The main purpose of this work is to study the scaling relations between fault length and displacement using a database that includes datasets of 21 faults with geometric data extracted from 3D seismic coherence cubes of the Norwegian Barents Sea. Multiple linear regression and Bayesian and Akaike information criterions are applied to obtain optimal regression parameters. Our dataset is unique since it includes segment lengths of individual faults, unlike the previously published datasets. Hence, we studied both the dependence of fault segment length and accumulated fault length on displacement. The latter relation (accumulated fault length versus displacement) shows a general agreement (positive correlation and power-law relation) with the previously published results that are mainly obtained from outcrop studies, although the slopes vary for different lithologies. The differences could be attributed to the unique characteristics of our dataset that includes data of all segment lengths of individual faults.
\end{abstract}

Keywords: fault attributes; seismic attributes; displacement; length; Akaike information criterion; Bayesian information criterion

\section{Introduction}

Fault geometric attributes such as fault displacement (D) and length (L) and their relations have been previously studied through statistical analysis of data coming from different data sources such as geological field studies, mines, interpreted seismic data, satellite images, and cores [1-7]. There are limitations inherent in almost all measuring methods. These limitations affect the statistical distribution of such data $[4,6]$. The main limitation with geological outcrops is accessibility. Outcrops are rarely fully three-dimensional and typically do not cover all scales of the fault population, but most of published data are still from outcrops [6,8]. For fault length measured along the strike, it is almost impossible to measure the total length of the large faults because of the accessibility problem in the field. Hence, what is usually measured is part of the fault dimension parallel to its dip direction (fault height) on the accessible vertical sections. Displacement can be measured in the field, although it also varies along the fault strike and dip, making assessment of entire displacement fields of individual faults difficult as exposures are limited. In addition, a fault is usually segmented, which means that the length of each single segment and the variation in displacement along the segment should be honored and contribute to the total length of the fault and its accumulated displacement. However, these details can be studied for seismic-scale faults using fault obtained from high-quality seismic cubes $[9,10]$. 
A compilation of previously published fault geometric data for different faults and rock types can be found in [6]. They found that the relation between fault geometric attributes cannot be defined by a single linear or power-law fit, and as the size (scale) of the faults change during their life-time, these relations might change. In other words, the slope of the power-law fit can change moving from smaller faults to more mature and larger faults, which interact with neighboring faults resulting in overlapping and linked faults. Kolyukhin and Torabi [7] examined the same compiled data. Their statistical study verifies that the relation between fault geometric attributes can change depending on the fault size.

Similar results were obtained, for example, in [11], where it was concluded that the relationship between fracture length and opening displacement cannot be described by a single scaling law. Further, Renshaw and Park [12] determined that there is a break in slope in the aperture-length scaling and showed the danger of applying universal scaling laws. A significant example of the evaluation of the scaling laws of fault size frequency distribution and the relation between fault length and displacement and their use for determination of total strain is presented in [13].

In the current study, we consider normal faults from the Norwegian Barents Sea, which occur predominantly in siliciclastic rocks with some minor intervals of carbonate rocks. The fault geometric data are extracted from 21 3D seismic cubes located in different parts of the Barents Sea, as shown in Figure 1 and Table 1. In order to increase fault detectability and make the data collection process more efficient and objective, the seismic cubes were converted to seismic coherence volumes. Seismic coherence is a seismic attribute, which quantifies the continuity of seismic reflectors in the vicinity of an analysis point [14]. Faults are better visualized on coherence attributes compared to standard seismic data. Fault traces are identified and picked from time slices in the coherence cubes, and displacement is measured from seismic lines oriented perpendicular to the fault traces. In the current study, we are able to extract the length of each fault segment of a single fault along its strike at different depths and measure fault displacements at several locations on each segment. Fault imaging using seismic attributes with the workflow we have used $[9,10]$ provides us with explicit images of faults. Extracting fault geometric data directly from such explicit images has less uncertainty compared to methods that incorporate interpretation of horizons and mapping horizon cutoffs since faults are directly extracted from seismic attributes with almost no interpretation [9].

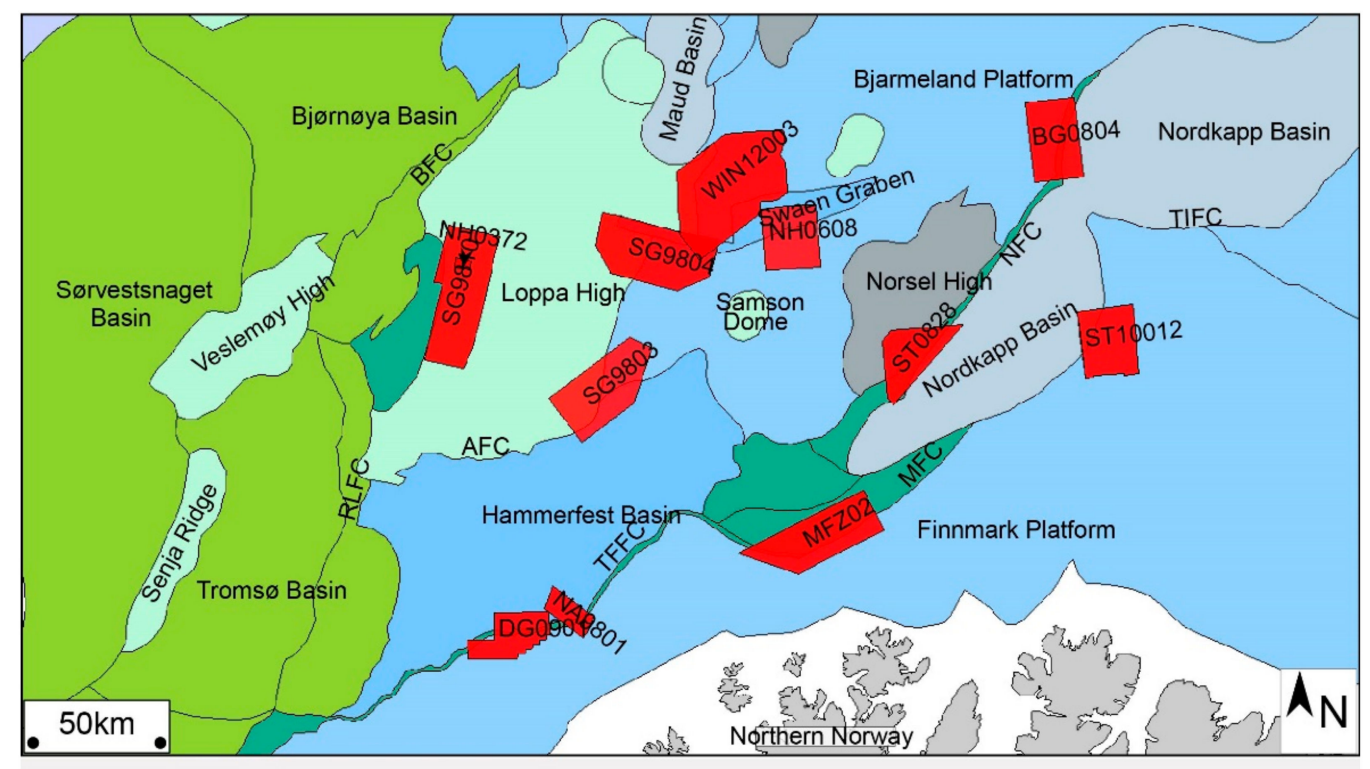

Figure 1. Map of the Norwegian Barents Sea with main structural elements (modified from Norwegian Petroleum Directorate (NPD) fact pages). Red polygons illustrate the location of seismic cubes. Light green, Cretaceous high; medium green, deep Cretaceous Basin; dark green, terraces and intra-basinal elevations. 
Table 1. Fault length (L), displacement (D), and estimated slope data.

\begin{tabular}{|c|c|c|c|c|c|c|}
\hline $\begin{array}{l}\text { Survey } \\
\text { Name }\end{array}$ & $\begin{array}{l}\text { Fault } \\
\text { Name }\end{array}$ & $\begin{array}{l}\text { Number of } \\
\text { Segments }\end{array}$ & $\begin{array}{l}\text { Number } \\
\text { of Depths }\end{array}$ & $b_{1}$ & $\begin{array}{c}\text { Maximum } \\
\text { Displacement (m) }\end{array}$ & $\begin{array}{c}\text { Maximum Accumulated } \\
\text { Length (m) }\end{array}$ \\
\hline NH608 & 1 & 63 & 25 & $1.11 \pm 0.29$ & 105.9 & 16,054 \\
\hline NH608 & 2 & 16 & 9 & $2.07 \pm 1.74$ & 20 & 3516.6 \\
\hline NH608 & 3 & 6 & 6 & $0.7 \pm 1.68$ & 13.2 & 1754.5 \\
\hline ST10012 & 4 & 78 & 14 & $0.51 \pm 0.38$ & 69 & 9581.1 \\
\hline ST10012 & 5 & 29 & 12 & $1.43 \pm 0.7$ & 27.6 & 4206.2 \\
\hline BG0804 & 6 & 44 & 18 & $0.12 \pm 0.57$ & 66.7 & 4342.3 \\
\hline SG9810 & 7 & 61 & 20 & $2.35 \pm 0.41$ & 30 & 8591.1 \\
\hline SG9810 & 8 & 14 & 9 & $1.95 \pm 0.88$ & 21 & 3045.1 \\
\hline SG9803 & 9 & 18 & 6 & $1.93 \pm 0.73$ & 22.7 & 7144.8 \\
\hline SG9804 & 10 & 46 & 17 & $1.18 \pm 0.48$ & 63.1 & 12,604 \\
\hline SG9804 & 11 & 5 & 5 & $0.67 \pm 1.03$ & 22.5 & 2757 \\
\hline NA9801 & 12 & 9 & 9 & $1.04 \pm 0.8$ & 20 & 1480.6 \\
\hline NA9801 & 13 & 3 & 3 & $0 \pm 2.88$ & 10.3 & 238.2 \\
\hline NA9801 & 14 & 3 & 3 & $1.25 \pm 5$ & 10.2 & 352.9 \\
\hline DG0901 & 15 & 7 & 5 & $1.42 \pm 1.9$ & 17.3 & 1566.8 \\
\hline ST0828 & 16 & 6 & 5 & $1.27 \pm 1.72$ & 43.6 & 2204.2 \\
\hline NH0372 & 17 & 6 & 6 & $0.42 \pm 0.41$ & 6 & 741.5 \\
\hline MFZ02 & 18 & 125 & 32 & $1.04 \pm 0.26$ & 53.3 & $16,721.6$ \\
\hline WIN12003 & 19 & 29 & 10 & $1.63 \pm 0.98$ & 63.1 & $23,255.7$ \\
\hline WIN12003 & 20 & 8 & 5 & $6.76 \pm 3.85$ & 17.2 & 7317.9 \\
\hline NA9801 & 21 & 6 & 4 & $0.7 \pm 2.28$ & 7 & 514.3 \\
\hline
\end{tabular}

The new fault geometric data extracted from seismic attribute volumes are used in the statistical analysis of L-D relation. For this analysis, we apply the Akaike information criterion (AIC) and the Bayesian information criterion (BIC), developed for a reasonable comparison of the statistical approximations with different numbers of model parameters [15]. This approach prevents the use of models with too many parameters. In [16], this approach was applied to study the dependence of fracture width on fracture length. Statistical analysis of the relationships between the main geometric fault attributes using the BIC was performed in [7]. In this paper, we consider the dependences of segment length and accumulated fault length on segment displacement for the selected normal faults from the Barents Sea (Figures 3-5). The technique used in this work allows extracting these characteristics from seismic data. In turn, it allows obtaining the new data for examination. A rigorous statistical analysis was applied to find the optimal regressions.

The paper is structured as follows: Section 2 describes in detail the methodology of fault data extraction from seismic data and statistical analysis; in Section 3 we describe the numerical results, and finally, the last section presents our conclusion.

\section{Methodology}

\subsection{Fault Data Extraction from Seismic Data}

The fault data used in this study were extracted from 3D seismic cubes in the Norwegian Barents Sea. The locations of the seismic surveys are shown in Figure 1. Coherence volumes were calculated from the seismic cubes using a structurally oriented semblance-based coherence algorithm (Figure 2), where coherence is computed along the local orientation of the seismic reflector [14,17]. We have mostly selected single and isolated faults in each seismic cube, however in some cases, there are neighboring faults that approach or link to the studied faults.

We extracted fault length data by manually digitizing fault traces (segments) from time slices in the seismic coherence volumes (Figure 2a,b). Each single fault contains many lateral segments at each depth, which are all captured within the 3D structure of the fault, extracted from seismic attributes (Figure 2b). The segment lengths of each fault will be used in this study. In addition, accumulated length has been provided, which corresponds to the sum of the fault segment lengths at each depth (Figure $2 b$ ). The vertical component of fault displacement was measured from seismic lines oriented perpendicular to the fault strike at several points along the digitized fault traces (Figure 2). 
We have not observed the strike slip component of the faults. Displacement measurements in this study are data-driven and involve no interpretation. For detailed information on the fault extraction and datasets used in this study, the readers are referred to [18]. The displacement measurements have been considered as throw and not true displacement. The measured displacements in time slices are throw and they were converted to meters using interval velocities from nearby wells. Each individual (single) fault in our database has a dataset, which contains all the lateral segments at different depths and the measured displacement at different points on the fault segments (Figure 2b).
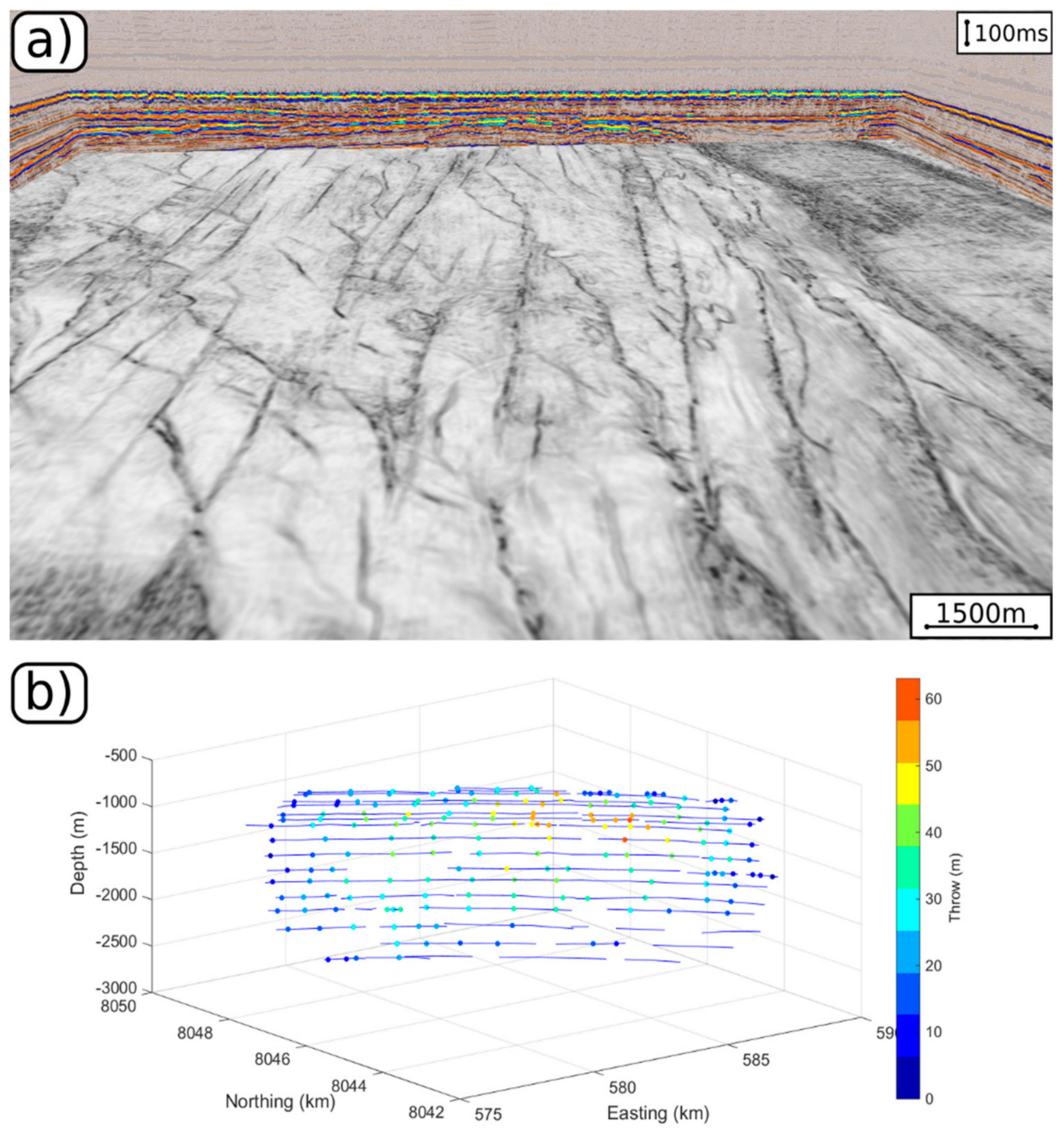

Figure 2. An example of a coherence time slice used to extract fault length data. Dark grey lines are fault segments (a). Digitized fault traces and displacement measurements, which are presented by color legend and circles on the fault segments from a single fault in the database (b).

\subsection{Statistical Analysis}

We study the relation between fault geometric attributes in logarithmic scale using multiple linear regressions. In the general case it has the form [19]

$$
y_{i}=b_{0}+\sum_{j=1}^{k} b_{j} \Phi_{j}\left(x_{i}\right)+\varepsilon_{i}=\gamma\left(x_{i}\right)+\varepsilon_{i}, i=1, \ldots, N
$$

where $\left\{x_{i}, y_{i}\right\}$ are the logarithm of the corresponding measured values of fault displacement and length, $b_{0}$ is the $y$-intercept value, $b_{j}$ is the slope of the $j$-th regression segment, $\Phi_{j}$ are $k$ predictor variables, $\varepsilon_{i}$ is the residual of the $i$-th measurement point, and $N$ is the number of measurements. Following $[7,16]$, 
we assume that the relation between fault geometric attributes can be described by a piecewise-linear function. For example, in the case of two slopes, this function has the form

$$
\gamma\left(x_{i}\right)=b_{0}+b_{1}\left[x_{i} I\left(x_{i}<x_{1}^{*}\right)+x_{1}^{*} I\left(x_{i} \geq x_{1}^{*}\right)\right]+b_{2}\left(x_{i}-x_{1}^{*}\right) I\left(x_{i} \geq x_{1}^{*}\right)
$$

where $I$ is a Heaviside indicator function and $x_{1}^{*}$ is the change-point between the two segments. Equation (2) can be simply extended to include a greater number of slopes $k$. In this work, we restrict the number of slopes to $k \leq 2$. The corresponding number of change-points $\left\{x_{i}^{*}\right\}$ would be $k-1$. In maximum likelihood estimation, the random residuals $\varepsilon_{i}$ are assumed independent and normally distributed with zero mean and standard deviation $\sigma$. Residuals normality was checked using a Jarque-Bera test [20].

Equations (1) and (2) allow us to get the best $\boldsymbol{b}$ and $\gamma$ for fixed changepoints. To determine the optimal number and positions of changepoints $x^{*}$ and to define the corresponding regression parameter $\boldsymbol{b}$, we apply the Bayesian information criterion (BIC). The BIC was developed in [21] to compare models with different number of parameters. This technique is based on the maximisation of the log-likelihood function (LLF) with an additional penalty term depending on the number of model parameters. We employ the modified BIC described in [16]:

$$
\operatorname{BIC}\left(y, x^{*}\right)=\operatorname{LLF}\left(y, x^{*}\right)-\frac{1}{2} \mathrm{q} \ln \left(\frac{N}{2 \pi}\right)
$$

where $\mathrm{q}=2 k+1$ is the total number of regression parameters $\left\{\boldsymbol{b}, \boldsymbol{x}^{*}, \sigma\right\}$ and $k$ is the number of slopes.

Along with BIC, other methods of model selection are also developed [15]. In this work, we compare the results obtained using the BIC with the results obtained using the Akaike information criterion (AIC)

$$
\operatorname{AIC}\left(y, x^{*}\right)=\operatorname{LLF}\left(y, x^{*}\right)-\mathrm{q} .
$$

Note that in the considered case the results of the AIC and BIC can only be different in optimal $k$ value.

For the same values of $k$, the AIC and BIC give the same regressions. The comparison of the AIC and BIC was performed, for example, in [22]. In general, their research has shown that in cases where these criteria give different results, it is preferable to use the BIC. Main et al. [16] favor the BIC when $N>46$.

\section{Results}

In this paper, we present results from the statistical analysis of the relation between fault length and displacement for the database that includes datasets of 21 normal faults, which encompasses many fault segments as part of the same fault at each depth (Figure 2b), and therefore we can investigate the effect of segmentation on the L-D relation. First, we consider the relationship between fault segment length and maximum displacement values (D) for all studied faults. We have also calculated the accumulated length (sum of the fault segment lengths at each depth) and studied its dependence on maximum displacement in order to compare our results with the previous data mainly from outcrop studies. In Table 1 we present the short description of considered data used to analyze the faults' geometric attributes.

\subsection{The Dependence of Segment Length on Displacement}

We first analyze the dependency of fault segment length on maximum displacement by using the multiple linear regression defined by Equation (1). In Figure 3 we present the studied data and optimal statistical approximation for $k=1$ which corresponds to the maximal BIC and AIC value. The optimal values of $k$ obtained by applying the BIC and AIC analysis are presented in Table 2. 
To show the dependence on the depth, the data points are colored according to the normalized depth of the measurement point $Z^{\prime}$ :

$$
Z^{\prime}=0.8(Z-\min (Z)) / \max (Z)-\min (Z),
$$

where $Z$ is the true depth. This normalization is applied in order to see if the plotted data point represents a shallow (blue) or deeper (red) point on the fault. The linear regression approximation presented in Figure 3 is

$$
y=0.95 x+4.71
$$

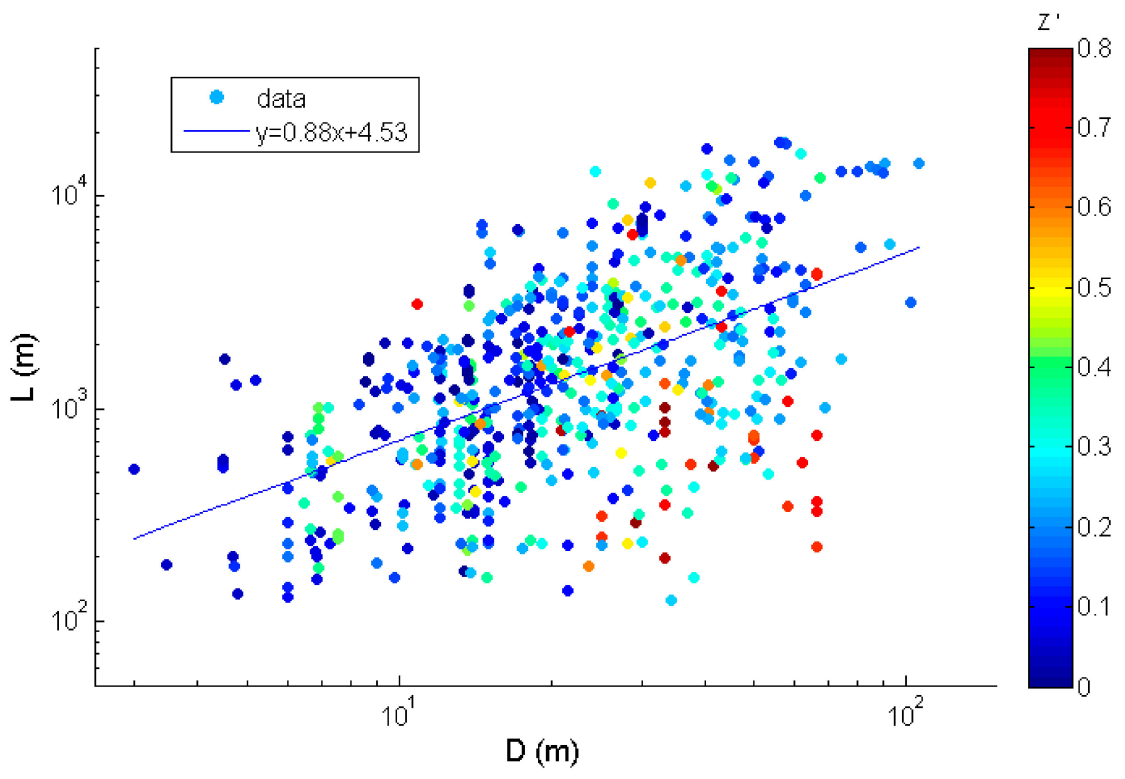

Figure 3. The dependence of all segment lengths on maximum displacement. The data points are colored according to the normalized depth of the measurement point $Z^{\prime}$.

In addition, from Figure 3 we can see that there is no link between the regression coefficient $b_{1}$ and the depth $Z$.

Following this study (Figure 3), we investigated the dependence $\ln L(\ln \mathrm{D})$ separately for segments of each of the 21 faults for $k=1$. The results for slopes $b_{1}$ and corresponding confidence intervals are presented in Table 1. For most considered faults, our statistical analysis showed rather wide confidence intervals (comparable to the maximum likelihood estimation itself) of the slope $b_{1}$. However, from Table 1 we can extract two sets of faults $\{4,6,17\}$ and $\{7,8,9,20\}$. The confidence intervals for these two sets are wide, but strictly disjoint $\left(b_{1}<1\right.$ and $\left.b_{1}>1\right)$. This means that the dependence of fault segment length on displacement could vary from fault to fault.

\subsection{The Dependence of Accumulated Length on Displacement}

The research presented in the previous section requires full information about the distribution of lengths of fault segments at each depth (Figure 2b). In this sub-section, we study the relation between accumulated fault length and maximum displacement. The results of the BIC analysis show that optimal statistical approximation is achieved for $k=2$, while the AIC shows that the optimal value of $k$ equals 1 (Table 2). The analyzed data extracted from seismic data for 21 studied faults and the preferred fit for $k=1$ (one slop model) and $k=2$ (solid lines) are illustrated in Figure 4. Obtained regressions are

$$
\begin{gathered}
y=1.23( \pm 0.13) x+4.36( \pm 0.43)(k=1), \\
x^{*}=45.4 ; b_{0}=3.87( \pm 0.51) ; b_{1}=1.41( \pm 0.16) ; b_{2}=0.19( \pm 0.61)(k=2) .
\end{gathered}
$$




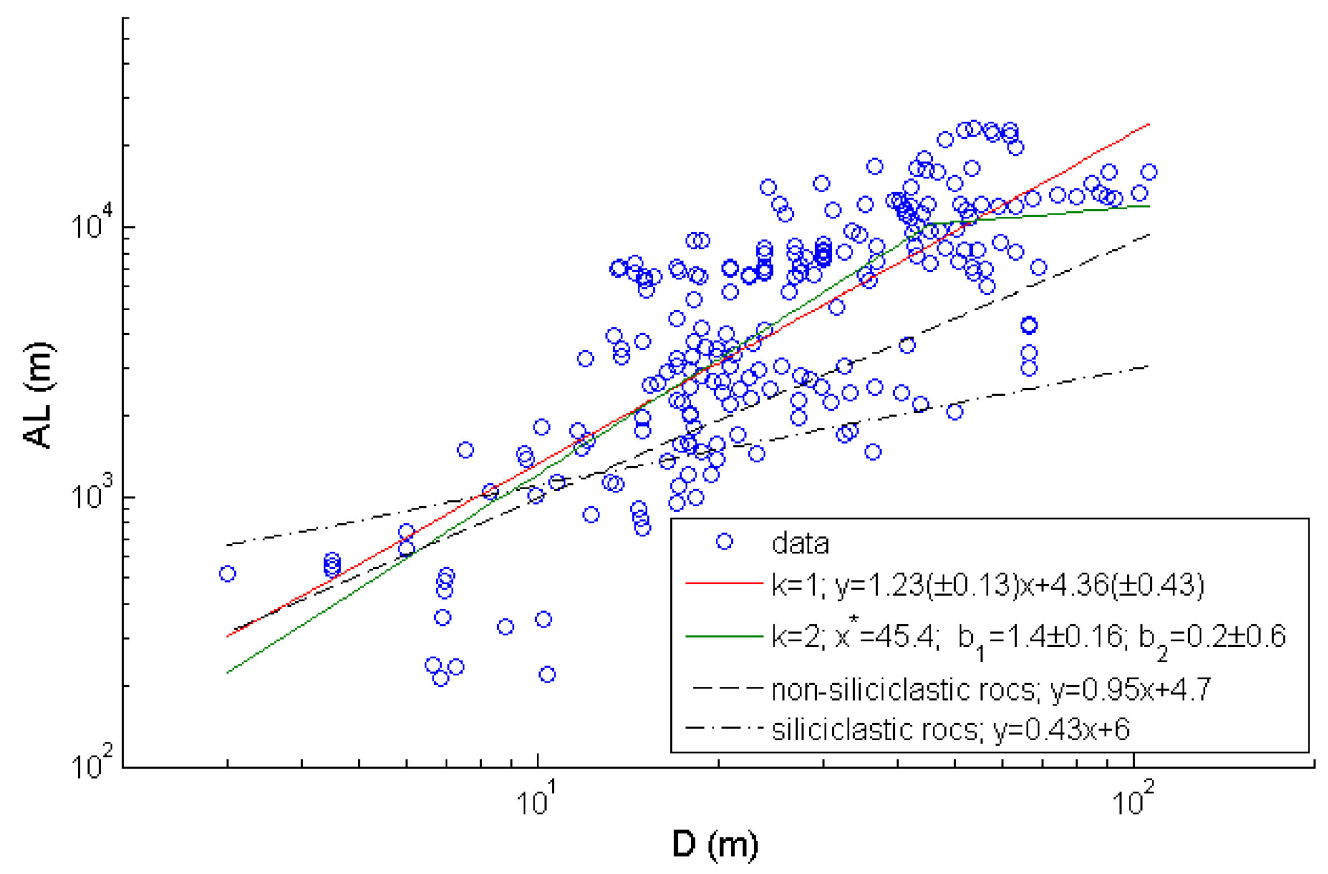

Figure 4. The dependence of fault accumulated length on maximum displacement (solid lines). Comparison with previous results for normal faults in siliciclastic and non-siliciclastic rocks (dashed and dash-dotted lines).

We also compare the results of the present study with results of a similar study for datasets of normal faults in siliciclastic and non-siliciclastic rocks previously considered in [7]. In this work, we perform the same analysis for this data in the shorter displacement range $1 \leq \ln (\mathrm{D}) \leq 5$, because this interval includes mainly large seismic faults. The regression parameters found for non-siliciclastic and siliciclastic rocks are

$$
\begin{gathered}
y=0.95( \pm 0.2) x+4.71( \pm 0.68)(\text { non }- \text { siliciclastic rocks }), \\
y=0.43( \pm 0.08) x+6.02( \pm 0.27) \text { (siliciclastic rocks). }
\end{gathered}
$$

The BIC indicates that in all considered cases the best statistical approximations are also linear regression with $k=1$ (dashed and dash-dotted lines in Figure 4). It is necessary to note that despite a smaller interval of $D$ values the statistical parameters of these curves are very close to the corresponding parameters found in [7]. On the other hand, for both considered lithologies, the line slopes differ from $b_{1}$ found in this study (solid line in Figure 4). This difference is more significant for siliciclastic rocks.

Finally, we study the dataset with only one value of maximum accumulated length and corresponding maximum displacement for each fault. These data could be more appropriate for comparison with previously published data. The maximal BIC value is achieved for $k=2$, but the BIC value for $k=1$ is rather close. The optimal value of $k$ obtained by using AIC equals 1 again (Table 2). Obtained regression function for $k=1,2$ are

$$
\begin{gathered}
y=1.33( \pm 0.48) x+3.96( \pm 1.53),(k=1) \\
x^{*}=32.1 ; b_{0}=2.65( \pm 2.11) ; b_{1}=1.84( \pm 0.75) ; b_{2}=0.25( \pm 1.34)(k=2) .
\end{gathered}
$$

The corresponding regression models are presented in Figure 5. For these relationships, we obtain a good quality of statistical approximation. In this study, we have only 21 data points and a rather wide confidence interval. Nevertheless, the slope $b_{1}$ in Figure 5 is much greater than the one found for previously published data for siliciclastic rocks $(0.43 \pm 0.08)$.

The comparison of BIC and AIC values for $k=1,2$ is presented in Table 2. 


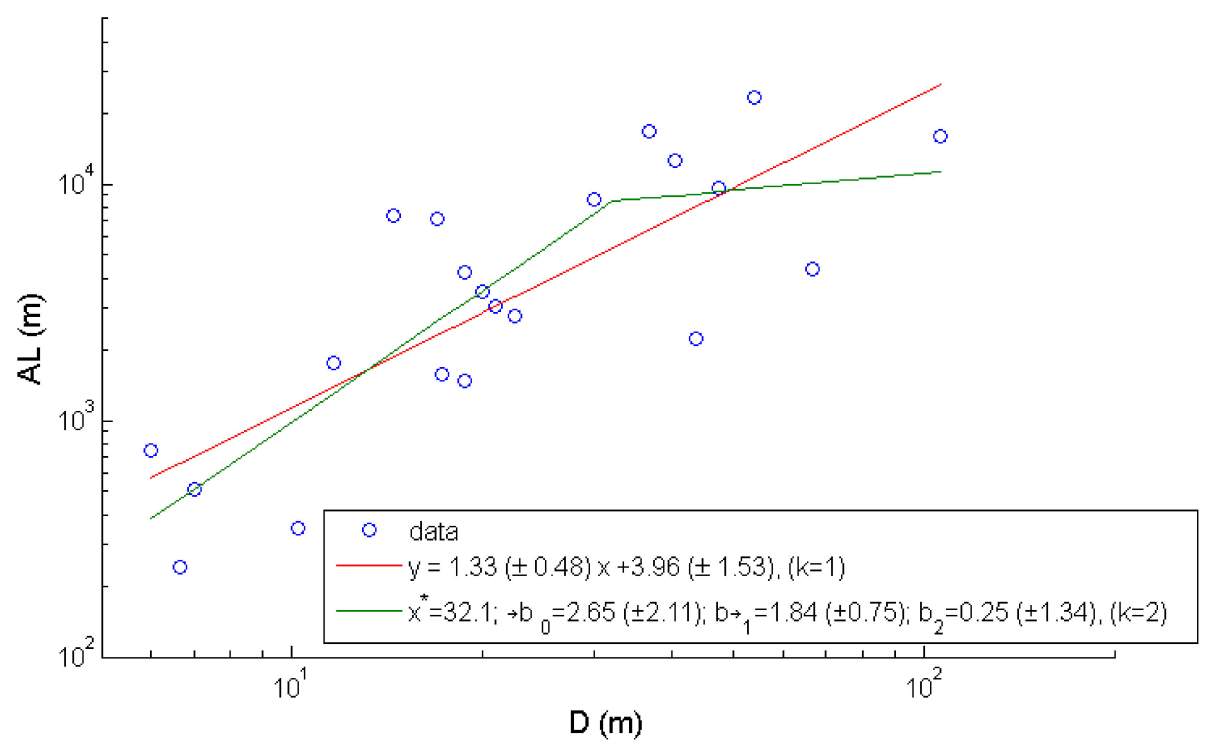

Figure 5. The dependence of maximal length on maximal displacement for 21 considered datasets $k=1$ and $k=2$.

Table 2. Comparison of Bayesian information criterion (BIC) and Akaike information criterion (AIC) values for $k=1,2$.

\begin{tabular}{cccc}
\hline & Figure 3 & Figure 4 & Figure 5 \\
\hline $\boldsymbol{N}$ & $\mathbf{5 8 2}$ & $\mathbf{2 2 3}$ & $\mathbf{2 1}$ \\
\hline $\mathrm{BIC}_{1}$ & -1.8041 & -515.30 & -27.99 \\
$\mathrm{BIC}_{2}$ & -1.8067 & -512.99 & -27.46 \\
$\mathrm{AIC}$ & -1.8003 & -512.94 & -29.18 \\
$\mathrm{AIC}_{2}$ & -1.8019 & -514.59 & -31.11 \\
\hline
\end{tabular}

\section{Discussion}

The fault segments were directly extracted from fault seismic attributes with almost no interpretation. The advantage of this approach is to derive fault geometric attributes from seismic data without the need for seismic interpretation. There are uncertainties related to seismic interpretation including using poor quality reflectors (usual for near fault locations) and interpolation and gridding challenges near fault plane locations. Poor reflector quality near faults is not an issue when we apply our method to image faults since we focus on discontinuity and not reflectors. Another advantage of this approach is the possibility to quickly generate a large amount of fault geometric data.

Using seismic attributes, we provide details of fault length and displacement in 3D [10]. This kind of data has not been previously studied in the datasets acquired mainly from outcrops. We have compared the results of this study with the previously published data for normal faults [10]. The accessibility of outcrop data is limited to 2D and 1D data from the field, in which the measurements are constrained by the size of the outcrop. This could explain the difference between the relations found in this study and the previous studies. When considering all the studied fault data (Figure 3), a power-law fits well to the relation between fault segment length and maximum displacement, although for the individual faults the relation does not hold some of the statistical conditions (Table 1). The comparison between the previously published data and our data (Figure 4) confirms a one slope power-law relation between fault length and maximum displacement in the range of the scale of the studied faults (seismic scale). Figure 5 includes the maximum accumulated length versus maximum displacement for the same depth. Hence, the number of data points for each fault has been reduced and only information from one part of the fault has been included. This scenario could be comparable with the part of a fault in outcrops with limited accessibility, although it does not give us the detailed 
structure of the fault in 3D. However, the power-law relation in Figures 3-5 indicates a mutual development of fault length and displacement for the seismic scale of faults.

For the statistical analysis of fault segment length dependence on maximum fault displacement, the optimal BIC approximation in logarithmic scale was achieved for one slope $(k=1)$. We have not observed any systematic influence of studied depth (indicated by the color) on the statistical dependence (Figure 3). For the dependence between accumulated fault length and maximum fault displacement, the maximal BIC values are achieved for $k=2$ (Figures 4 and 5). For all data analyzed in Figures $3-5$, the AIC shows that optimal $k$ is equal to 1 , which means that it is more suitable to use simpler linear regression (Table 2). Following [13,22], we can estimate that it is preferable to apply the BIC for datasets with large values $N$ (data presented in Figures 3 and 4). For the dataset considered in Figure $5, N=21$, hence we suggest the regression obtained for $k=1$ because of rather close BIC values for $k=1$ and $k=2$. For the same $k$ all regression parameters are the same for both the AIC and BIC.

The maximum likelihood estimation and Bayesian information criterion described by Equations (1) and (3) require the independence and normality of residuals $\varepsilon_{i}$. In general, these conditions are fulfilled in the presented study. An exception is a common consideration of segments for all the faults presented in Figure 3, where there is a problem with the residuals independence condition. This makes it difficult to use the BIC and standard estimation of confidence intervals, but the least-squares estimate of the value of $b_{1}$ remains correct [19].

\section{Conclusions}

The following conclusions can be made from this study:

1. Utilizing seismic coherence attributes, we are able to extract segment lengths of a single fault at different depths.

2. All considered L-D relations are analyzed using the Bayesian information criterion and Akaike information criterion. In all considered cases, statistical analysis using the AIC shows that optimal statistical approximation in logarithmic scale is the simple linear regression $(k=1)$, whereas optimal statistical approximation for the dependence of accumulated length on displacement obtained using the BIC is achieved for $k=2$.

3. L-D relation for individual segment length versus maximum displacement significantly differs for different faults.

4. When comparing our result with the analysis of previously published data, in general, they are in qualitative agreement. However, the slope value is greater for the new data. This difference could be related to including detailed measurements from each fault segment in the current datasets.

Author Contributions: Conceptualization, A.T., B.A.; methodology, B.A., D.K.; software, A.L., D.K., and T.K.; data analysis, A.L., D.K., and T.K.; writing-original draft preparation, D.K.; writing-review and editing, D.K., A.T., A.L., and B.A.; project administration, D.K., A.T.

Funding: This research was funded by the NORRUSS and Petromaks2 programs of the Norwegian Research Council (project 243628) and the Russian Foundation for Basic Research (grant no. 15-55-20004).

Conflicts of Interest: The authors declare no conflicts of interest.

\section{References}

1. Clark, R.M.; Cox, S.J. A modern regression approach to determining fault displacement- length scaling relationships. J. Struct. Geol. 1996, 18, 147-152. [CrossRef]

2. Watterson, J.; Walsh, J.J.; Gillespie, P.A.; Easton, S. Scaling systematics of fault sizes on a large scale range fault map. J. Struct. Geol. 1996, 18, 199-214. [CrossRef]

3. Walsh, J.J.; Nicol, A.; Childs, C. An alternative model for the growth of faults. J. Struct. Geol. 2002, 24, 1669-1675. [CrossRef]

4. Kim, Y.-S.; Sanderson, D.J. The relationship between displacement and length of faults. Earth-Sci. Rev. 2005, 68, 317-334. [CrossRef] 
5. Schultz, R.A.; Soliva, R.; Fossen, H.; Okubo, C.H.; Reeves, D.M. Dependence of displacement-length scaling relations for fractures and deformation bands on the volumetric changes across them. J. Struct. Geol. 2008, 30, 1405-1411. [CrossRef]

6. Torabi, A.; Berg, S. Scaling of fault attributes: A review. Mar. Petrol. Geol. 2011, 28, 1444-1460. [CrossRef]

7. Kolyukhin, D.; Torabi, A. Statistical analysis of the relationships between faults attributes. J. Geophys. Res. 2012, 117, B05406. [CrossRef]

8. Childs, C.; Manzocchi, T.; Walsh, J.J.; Bonson, C.G.; Nicol, A.; Schöpfer, M.P.J. A geometric model of fault zone and fault rock thickness variations. J. Struct. Geol. 2009, 31, 117-127. [CrossRef]

9. Libak, A.; Alaei, B.; Torabi, A. Fault visualization and identification in fault seismic attribute volumes: Implications for fault geometric characterization. Interpretation 2017, 5, B1-B16. [CrossRef]

10. Torabi, A.; Alaei, B.; Kolyukhin, D. Analysis of fault scaling relations using fault seismic attributes. Geophys. Prospect. 2017, 65, 581-595. [CrossRef]

11. Hatton, C.G.; Main, I.G.; Meredith, P.G. Non-universal scaling of fracture length and opening displacement. Nature 1994, 367, 160-162. [CrossRef]

12. Renshaw, C.E.; Park, J.C. Effect of mechanical interactions on the scaling of fracture length and aperture. Nature 1997, 386, 482-484. [CrossRef]

13. Scholz, C.H.; Cowie, P.A. Determination of geologic strain from fault slip data. Nature 1990, 346, 837-839. [CrossRef]

14. Chopra, S.; Marfurt, K.J. Seismic attributes for prospect identification and reservoir characterization. SEG Geophys. Dev. Ser. 2007. [CrossRef]

15. Bulteel, K.; Wilderjans, T.F.; Tuerlinckx, F.; Ceulemans, E. CHull as an alternative to AIC and BIC in the context of mixtures of factor analyzers. Behav. Res. Methods 2013, 45, 782-791. [CrossRef] [PubMed]

16. Main, I.G.; Leonard, T.; Papasouliotis, O.; Hatton, C.G.; Meredith, P.G. One slope or two? Detecting statistically significant breaks of slope in geophysical data, with application to fracture scaling relationships. Geophys. Res. Lett. 1999, 26, 2801-2804. [CrossRef]

17. Marfurt, K.J.; Kirlin, R.L.; Farmer, S.L.; Bahorich, M.S. 3-D seismic attributes using a semblance-based coherency algorithm. Geophysics 1998, 63, 1150-1165. [CrossRef]

18. Torabi, A.; Alaei, B.; Libak, A. Normal fault 3D geometry and displacement revisited: Insights from faults in the Norwegian Barents Sea. Mar. Petrol. Geol. 2019, 99, 135-155. [CrossRef]

19. Johnson, R.A.; Wichern, D.W. Applied Multivariate Statistical Analysis, 6th ed.; Pearson Prentice Hall: Upper Saddle River, NJ, USA, 2007; p. 773. ISBN 0131877151.

20. Jarque, C.M.; Bera, A.K. A test for normality of observations and regression residuals. Int. Stat. Rev. 1987, 55, 1-10. [CrossRef]

21. Schwarz, G. Estimating the dimension of a model. Ann. Stat. 1978, 6, 461-464. [CrossRef]

22. Koehler, A.B.; Murphree, E.S. A Comparison of the Akaike and Schwarz Criteria for Selecting Model Order. J. R. Stat. Soc. Ser. C (Appl. Stat.) 1988, 37, 187-195. [CrossRef]

(c) 2018 by the authors. Licensee MDPI, Basel, Switzerland. This article is an open access article distributed under the terms and conditions of the Creative Commons Attribution (CC BY) license (http://creativecommons.org/licenses/by/4.0/). 